\title{
Condoms in the prevention of HIV and AIDS
}

\section{Lucian Jayasuriya ${ }^{1}$}

Within a quarter of a century HIV/AIDS has devastated mankind.

It is the most critical health issue facing mankind today. AIDS is the leading cause of death in Africa and the fourth leading cause of death worldwide. Millions of children have been orphaned especially in Africa.

What are the strategies available to control this scourge?

There is no vaccine in sight. Treatment with highly active anti retroviral therapy only prolongs life and prevents mother to child transmission to some extent..

Prevention remains the answer to the problem.

Theoretically we say prevention is by $\mathrm{ABC}-$ abstinence, be faithful and condoms. We mouth the first two and depend on condom promotion to prevent HIV transmission. This strategy has obviously failed because the numbers getting infected are increasing continuously. Sadly however, those who lead the campaign to control HIV/AIDS internationally do not seem to want to face reality. They seem to want to have the cake and eat it.
They want to promote sexual activity as well as control the spread of sexually transmitted infections.

To prevent transmission of HIV by sexual intercourse condoms of good quality have to be used correctly and consistently. This is the crunch. It is obvious that these criteria of usage are often not fulfilled. The end result is that the condom usage results in acquiring infection sooner or later. Therefore condom promotion should not be a strategy for prevention of HIV transmission.

The pandemic in Africa is due to the fact that sex before marriage and sex after marriage are considered as the norm in these cultures.

Sri Lankan culture does not consider pre- marital and extra marital sex as the norm. This may a reason that Sri Lanka remains a low prevalence country for HIV/AIDS.

Abstinence before marriage and being faithful in marriage should be promoted to keep our low prevalence status. Usage of condoms should not be promoted except to commercial sex workers and men who have sex with men.

\section{The first intravenous drug user with HIV infection in Sri Lanka}

\author{
MPW Gunathilake1, A Wijewickrama², S Mananwatte ${ }^{3}$, S Samarakoon ${ }^{4}$
}

\section{Introduction}

Blood to blood transmission of HIV either by needle sharing intravenous drug use or blood transfusion is much more effective than sexual transmission.

Explosive epidemics of HIV, related to intravenous drug use have been observed in many parts of the world.

Injecting drug use is not reported as a problem in Sri Lanka, at present. The National Dangerous Drugs Control Board estimates 40,000 heroin users in the country and
200,000 cannabis users, only $2 \%$ are estimated to be injecting drug users. While the World Bank estimates $7.5 \%$ of the illicit drug users are injectors. ${ }^{1}$

We are reporting the first intravenous drug user with HIV infection in this country.

\section{Case Report}

In July 2004, HIV-2 infection was detected in a 36 year old Middle East returnee. He admitted to have been an intravenous drug user since 1992, from the time he was

${ }^{1}$ Senior Register in Venereology, Central STD Clinic National STD/AIDS Control Programme, ${ }^{2}$ Consultant Physician, Infectious diseases Hospital, Gothatuwa, ${ }^{3}$ Consultant Microbiologist, ${ }^{4}$ Consultant Venereologist, Central STD Clinic, National STD/AIDS Control Programme. 